Revista Brasil. Bot., V.25, n.4, p.469-474, dez. 2002

\title{
Influência de fatores ambientais sobre o crescimento in vitro de Hypnea musciformis (Wulfen) Lamouroux (Rhodophyta)
}

\author{
ISOLDA C. BRAVIN ${ }^{1}$ e YOCIE YONESHIGUE-VALENTIN ${ }^{1,2}$
}

(recebido: 31 de outubro de 2001; aceito: 31 de julho de 2002)

\begin{abstract}
The influence of environmental factors on in vitro growth of Hypnea musciformis (Wulfen) Lamouroux (Rhodophyta)). Hypnea musciformis (Wulfen) Lamouroux (Hypneaceae, Gigartinales) is an important carrageenan resource of Brazil used in the industry of phycocolloid gelling agent. The objective of this study was to investigate the influence of temperature $\left(20^{\circ} \mathrm{C}\right.$ and $\left.25^{\circ} \mathrm{C}\right)$, enriched seawater with Provasoli's solution and aeration on the in vitro development of this species. Thalli of H. musciformis were collected from Prainha, Arraial do Cabo (RJ) in two different seasons of the year 2000 (April and July). In the laboratory, apical fragments weighing $0.2 \mathrm{~g}(15 \mathrm{~mm}$ long) were excised from the thalli and prepared for incubations at different abiotic factors. In decreasing order, multifactorial analysis of variance showed that the factors of higher influence on the growth of fragments were: water nutrient enrichment, aeration, temperature and season. All fragments increased in biomass and showed high phenotypic plasticity. The best results were obtained in nutrient enriched medium, aeration and at $25{ }^{\circ} \mathrm{C}$ (biomass of $17 \mathrm{~g}$ and specific growth rate of $20.79 \%$.day ${ }^{-1}$ ). On the other hand, $\mathrm{H}$. musciformis did not respond well in non nutrient enriched medium, absence of aeration and at $25^{\circ} \mathrm{C}$ (biomass of $0.7 \mathrm{~g}$ and specific growth rate of $0.07 \%$.day $^{-1}$ ). The thalli collected in different seasons of the year did not demonstrate any sharp differences. We concluded that cultivation of apical fragments of $\mathrm{H}$. musciformis produces good results that can provide a basis for future applications.
\end{abstract}

RESUMO - (Influência de fatores ambientais sobre o crescimento in vitro de Hypnea musciformis (Wulfen) Lamouroux (Rhodophyta)). Hypnea musciformis (Wulfen) Lamourox (Hypneaceae, Gigartinales) é uma fonte importante para o Brasil de um agente ficocolóide geleificante (carragenano) usado na indústria. O objetivo deste estudo foi o de investigar a influência da temperatura $\left(20\right.$ e $\left.25^{\circ} \mathrm{C}\right)$, do enriquecimento água do mar com a solução de Provasoli e aeração sobre o desenvolvimento desta espécie in vitro. Os talos de H. musciformis foram coletados na Prainha, Arraial do Cabo (RJ) em duas diferentes épocas do ano de 2000 (abril e julho). No laboratório, fragmentos apicais pesando $0,2 \mathrm{~g}$ ( $15 \mathrm{~mm}$ de comprimento) foram excisados do talo e preparados para as incubações em diferentes combinações de fatores. Em ordem decrescente, a análise de variância multifatorial mostrou que os fatores de maior influência sobre o crescimento dos fragmentos apicais dos talos de H. musciformis foram: enriquecimento, aeração, a temperatura e o período do ano. Todos os fragmentos tiveram aumento de biomassa mostrando plasticidade fenotípica durante o período experimental. Os melhores resultados foram obtidos em culturas com meio de enriquecimento, com aeração e a $25^{\circ} \mathrm{C}$ (biomassa de $17 \mathrm{~g}$ e taxa específica de crescimento de $20,79 \%$.dia ${ }^{-1}$ ). Por outro lado, H. musciformis não respondeu bem em culturas não enriquecidas, ausência de aeração e a $25^{\circ} \mathrm{C}$ (biomassa de 0,7 e taxa de crescimento de $0,07 \% \cdot$ dia $^{-1}$ ). As plantas cultivadas nas duas épocas do ano não demonstraram diferenças significativas. Concluí-se que a partir dos fragmentos apicais de H. musciformis cultivados, obteve-se bons resultados que fornecerão uma base para futuras aplicações.

Key words - aeration, culture, enrichment, Hypnea musciformis, temperature

\section{Introdução}

Hypnea musciformis (Wulfen) Lamouroux é uma espécie de grande interesse econômico, pois é produtora de k-carragenano, polissacarídeo com alta qualidade espessante, possuindo ainda substâncias com potencial farmacêutico, as quais têm atividade antiviral (Santos et al. 1999), vermífuga, anti-inflamatória e mio-relaxante (Schenkman 1989).

Esta espécie é amplamente distribuída no litoral brasileiro, embora tenha havido declínio do estoque

\footnotetext{
1. Universidade Federal do Rio de Janeiro, Instituto de Biologia, Departamento de Botânica, Av. BrigadeiroTrompowsky s/n. Ilha do Fundão, 21941-900 Rio de Janeiro, RJ, Brasil.

2. Autor para correspondência: yocie@biologia.ufrj.br
}

natural graças a alta exploração (Oliveira-Filho 1981, 1998). A preocupação com o esgotamento da matéria algácea de $H$. musciformis no ambiente, levou a publicação de alguns trabalhos que avaliam a produção de biomassa em vários locais do mundo, incluindo o Brasil (Schenkman 1989, Wallner et al. 1992, Berchez et al. 1993, Reis \& Yoneshigue-Valentin 1998, Faccini \& Berchez 2000), nos Estados Unidos (Durako \& Dawes 1980), na Índia (Rao 1970) e no Havaí (Mshigeni 1977). Além disso, estudos sobre o cultivo no mar também foram realizados, com a finalidade de diminuir a exploração de populações naturais (Mshigeni 1976, Berchez et al. 1989, Berchez \& Oliveira-Filho 1989, Wallner et al. 1992, Berchez et al. 1993).

Berchez et al. (1993) observaram que o movimento da água foi um fator determinante no controle do 
crescimento de Hypnea musciformis em substratos artificiais ancorados sobre cordões lineares. Reis \& Yoneshigue-Valentin (1998) constataram também que o movimento da água foi um dos principais fatores ambientais, seguido pela insolação, com forte interferência sobre a produção de biomassa das populações no estado do Rio de Janeiro.

Embora o controle da variação de fatores abióticos, como a irradiância, a temperatura, a salinidade, os nutrientes e a movimentação da água, seja mais viável no laboratório, no Brasil, são poucos os trabalhos publicados que utilizam desses fatores como ferramenta para detectar diferenças no cultivo de H. musciformis (Yokoya \& Oliveira 1992a, 1992b).

O objetivo do presente estudo foi o de avaliar a influência da temperatura, do enriquecimento da água do mar e do movimento da água sobre o desenvolvimento de $H$. musciformis, coletada em região de ressurgência (Arraial do Cabo, RJ) e o de estabelecer as condições de cultivo que sejam apropriadas para obtenção de uma maior biomassa.

\section{Material e métodos}

Os talos de Hypnea musciformis (Wulfen) Lamouroux em fase não reprodutiva foram coletados em abril e julho de 2000 na enseada da Prainha, localizada no Município de Arraial do Cabo, RJ (22 $57^{\prime} 30^{\prime}$ 'S e $42^{\circ} 01^{\prime} 30^{\prime}$ W), região sob influência do fenômeno da ressurgência, mais freqüente entre os meses de setembro e fevereiro (Yoneshigue-Valentin \& Valentin 1992). Imediatamente após a coleta fragmentos selecionados de talos saudáveis foram lavados com água do mar e transportados em garrafas térmicas até o laboratório.

Após seleção, os talos foram lavados cinco vezes em água do mar filtrada com membrana celulósica Millipore de $0,45 \mu \mathrm{m}$ de poro para retirada de materiais indesejáveis, como epífitas, grãos de areia e pequenos animais. A água do mar utilizada como base para os meios de cultura foi proveniente de Arraial do Cabo (salinidade de 35,6 u.p.s. e pH de 8,0). Esta água do mar foi filtrada e enriquecida com a solução de nutrientes e vitaminas denominada PES (Provasoli 1968) com salinidade de 35,6 u.p.s. e pH de 7,8. Três fragmentos apicais com gavinhas $(0,2 \mathrm{~g})$, medindo cerca de $15 \mathrm{~mm}$ de comprimento e $500 \mu \mathrm{m}$ de diâmetro foram excisados do talo e colocados em frascos do tipo Erlenmeyer contendo $400 \mathrm{~mL}$ de água do mar com ou sem PES. Foram testadas temperaturas de 20 e $25{ }^{\circ} \mathrm{C}$. Todas as réplicas foram submetidas a irradiância constante de $30 \pm 5 \mu \mathrm{mol} \cdot \mathrm{m}^{-2} \cdot \mathrm{s}^{-1}$ (duas lâmpadas Osram, luz do dia, dispostos lateralmente em relação ao frasco de incubação) sob fotoperíodo de 12:12 h. A irradiância foi medida através de um quantâmetro do tipo Licor LI 1000 Data Logger, conectado a um sensor quântico LI-190SA. No sentido de determinar se a aeração da água aumenta a produção de biomassa dessa planta, foi inserido no meio de cultura um tubo de plástico esterilizado ligado a uma bomba de aquário. Culturas estagnadas, como controle, também fizeram parte deste estudo.

Foram preparadas três repetições de cada tratamento que consiste nas combinações utilizando as temperaturas de $20{ }^{\circ} \mathrm{C}$ e $25^{\circ} \mathrm{C}$, meio enriquecido (CE) ou não (SE) e culturas aeradas (CA) ou não (SA).

Os experimentos tiveram duração de 40 dias, com trocas do meio de cultura a cada 10 dias, quando também foi avaliado o crescimento da planta através de massa úmida. Taxas de crescimento específico foram calculadas utilizando a seguinte fórmula (Yoneshigue-Braga \& Neves 1981):

taxa de crescimento $\left(\% \cdot \mathrm{d}^{-1}\right)=1 . \mathrm{t}^{-1}\left(\mathrm{Ln} \mathrm{Wt} . \mathrm{Wo}^{-1}\right) \mathrm{X} 100 \%$,

onde, Wo e Wt são o peso inicial e final, respectivamente, e t é o período da incubação em dias

Para verificar a influência dos fatores ambientais testados (temperatura, enriquecimento, aeração e época do ano) e a importância relativa de cada fator, os resultados foram avaliados através de análise de variância multifatorial, utilizando o programa Statistica (StatSoft, v.5.1).

\section{Resultados}

Os talos de Hypnea musciformis apresentaram crescimento expressivo no cultivo realizado sob todas as condições propostas (figuras 1,2). As curvas de crescimento (figura 1) demonstram maior ganho de massa úmida para plantas cultivadas com a adição de nutrientes e vitaminas (CE), sob as duas condições de aeração (CA e SA) e nas duas temperaturas testadas $\left(20\right.$ e $\left.25{ }^{\circ} \mathrm{C}\right)$. No entanto, maior ganho de biomassa pode ser observado em plantas cultivadas a $25^{\circ} \mathrm{C}$, com a agitação do meio de cultura (cerca de 85 vezes, $16,9 \mathrm{~g}$, o peso inicial) (figura $1 \mathrm{~A}$ ). As culturas incubadas a $20^{\circ} \mathrm{C}, \mathrm{CA}$ e $\mathrm{CE}$, também alcançaram valores altos de massa úmida ao final do cultivo $(15,8 \mathrm{~g})$. Os tratamentos $\mathrm{SE}$, a $25^{\circ} \mathrm{C}$ e $20^{\circ} \mathrm{C}$ e CA (figura 1B) revelaram um crescimento maior ao final dos 40 dias (respectivamente, 1,05 g e 1,24 g) quando comparados às demais condições sem enriquecimento. As plantas cultivadas em meio sem enriquecimento apresentaram coloração amarelada à rósea. Em SA, constatou-se após o período de 40 dias uma forte queda do crescimento, observada em CE e SE, tanto a $20^{\circ} \mathrm{C}$ (respectivamente, $2,0 \mathrm{~g}$ e $0,7 \mathrm{~g}$ ), quanto para $25^{\circ} \mathrm{C}(0,7 \mathrm{~g})$. No tratamento com adição de meio nutritivo (CE), após o vigésimo dia de cultivo, observou-se a degeneração dos fragmentos cultivados a $25{ }^{\circ} \mathrm{C}$, que alcançaram massa final de cinco vezes a inicial $(1 \mathrm{~g})$. 
A

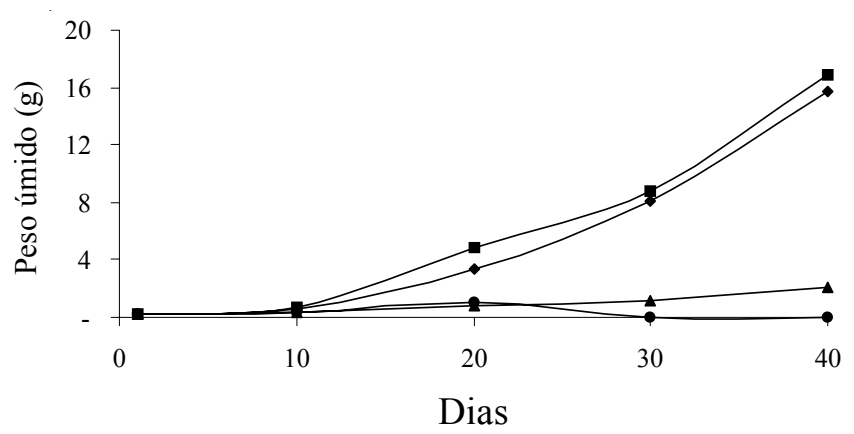

B

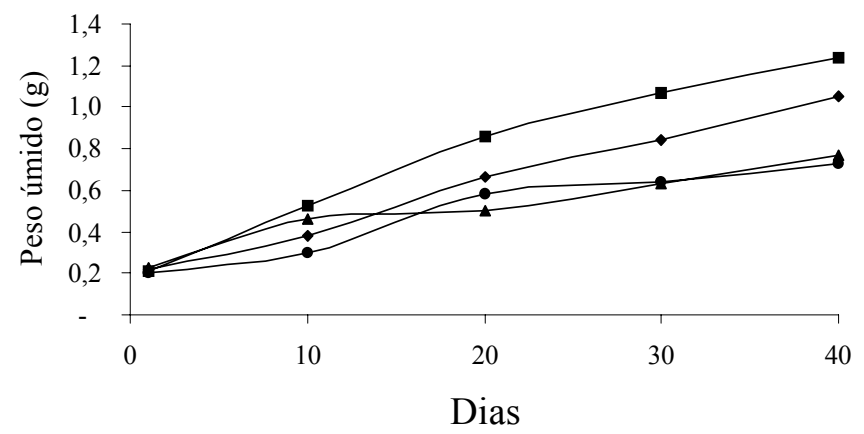

Figura 1. Curvas de crescimento de exemplares de Hypnea musciformis coletados no mês de abril de 2000 cultivados in vitro durante o período de 40 dias com a adição de PES (A) e sem a adição (B). $\bullet 20^{\circ} \mathrm{C}$, com aeração, $-25^{\circ} \mathrm{C}$, com aeração, $\boldsymbol{\wedge}$ $20{ }^{\circ} \mathrm{C}$, sem aeração e $\bullet 25^{\circ} \mathrm{C}$, sem aeração.

O material coletado em abril, cultivado a $25^{\circ} \mathrm{C}$ (CA) apresentou maior taxa de crescimento específico principalmente nos primeiros 20 dias de cultivo, quando comparada àquele crescido a $20{ }^{\circ} \mathrm{C}$ nesse mesmo período (tabela 1). As plantas coletadas em julho comportaram-se de forma semelhante às de abril quanto ao padrão de crescimento em todas as condições de cultivo testadas (figura 2). Ocorreu também crescimento mais satisfatório em culturas incubadas com enriquecimento (figura $2 \mathrm{~A}$ ). Entretanto, quando comparado ao crescimento das plantas de abril, pode-se observar que as plantas cultivadas a 20 e $25{ }^{\circ} \mathrm{C}$, em CE e CA (figura $2 \mathrm{~A}$ ), atingiram menor valor de massa úmida (respectivamente, 51 e 67 vezes o peso inicial). As taxas de crescimento em julho, quando confrontadas com as de abril, nos primeiros dez dias de cultivo foram maiores para quase todas as combinações de fatores testadas, sendo a maior para culturas incubadas a $25^{\circ} \mathrm{C}$, CA e CE. No entanto, depois desse período, as taxas cairam bruscamente (tabela 1). Comparando-se com as de abril, essas taxas decresceram acentuadamente somente após o vigésimo dia de cultivo.

A análise de variância multifatorial (tabela 2) revelou influência significativa $(\mathrm{p}<0,00001)$ de todos os fatores estudados, sendo por ordem de importância: o enriquecimento, a aeração, a temperatura e, por fim, a época do ano.

Tabela 1. Média das taxas de crescimento $\left(\% \cdot \mathrm{dia}^{-1}, \mathrm{n}=3\right)$ de exemplares de Hypnea musciformis coletados nos meses de abril e julho de 2000 cultivados in vitro durante o período de 40 dias nas temperaturas de $20^{\circ} \mathrm{C}$ e $25^{\circ} \mathrm{C}$, com ou sem aeração e com (CE) ou sem (SE) o enriquecimento de Provasoli (PES) (- : a cultura não sobreviveu).

\begin{tabular}{|c|c|c|c|c|c|c|c|c|}
\hline \multirow[t]{4}{*}{ Dias } & \multicolumn{8}{|c|}{ Taxas de crescimento } \\
\hline & \multicolumn{4}{|c|}{$20^{\circ} \mathrm{C}$} & \multicolumn{4}{|c|}{$25^{\circ} \mathrm{C}$} \\
\hline & \multicolumn{2}{|c|}{ Com aeração (CA) } & \multicolumn{2}{|c|}{ Sem aeração (SA) } & \multicolumn{2}{|c|}{ Com aeração (CA) } & \multicolumn{2}{|c|}{ Sem aeração (SA) } \\
\hline & $\mathrm{CE}$ & SE & $\mathrm{CE}$ & SE & $\mathrm{CE}$ & SE & $\mathrm{CE}$ & SE \\
\hline \multicolumn{9}{|l|}{ Abril } \\
\hline 10 & 10,81 & 5,47 & 7,44 & 6,93 & 11,89 & 9,26 & 4,52 & 4,06 \\
\hline 20 & 8,99 & 2,76 & 3,71 & 0,42 & 9,80 & 2,42 & 5,49 & 3,30 \\
\hline 30 & 2,91 & 0,80 & 1,08 & 0,77 & 1,94 & 0,73 & - & 0,33 \\
\hline 40 & 1,67 & 0,56 & 1,47 & 0,50 & 1,64 & 0,37 & - & 0,33 \\
\hline \multicolumn{9}{|l|}{ Julho } \\
\hline 10 & 14,57 & 6,93 & 8,68 & 6,19 & 20,79 & 8,75 & 11,15 & 6,93 \\
\hline 20 & 4,87 & 1,47 & 2,35 & 1,98 & 4,14 & 1,03 & 2,05 & 1,85 \\
\hline 30 & 2,92 & 1,10 & 0,81 & 1,03 & 2,82 & 1,06 & - & 0,71 \\
\hline 40 & 1,53 & 0,65 & 1,99 & 0,78 & 1,24 & 0,50 & - & 0,07 \\
\hline
\end{tabular}



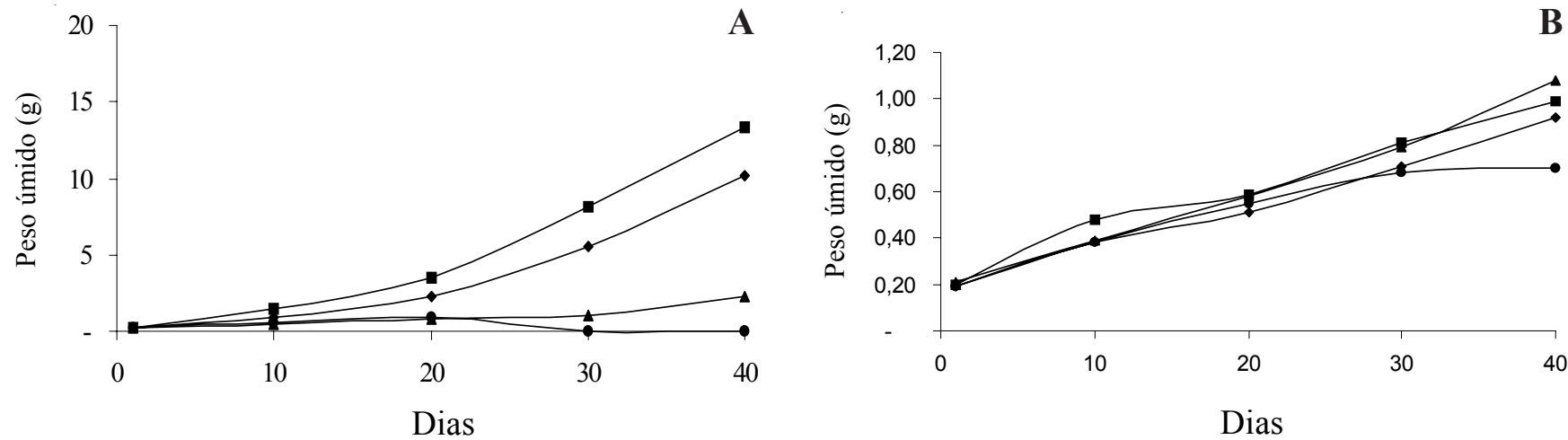

Figura 2. Curvas de crescimento de exemplares de Hypnea musciformis coletados no mês de julho de 2000 cultivados in vitro durante o período de 40 dias com a adição de PES (A) e sem a adição (B). $\bullet 20^{\circ} \mathrm{C}$, com aeração, $-25^{\circ} \mathrm{C}$, com aeração, $\boldsymbol{\Delta}$ $20^{\circ} \mathrm{C}$, sem aeração e $\bullet 25^{\circ} \mathrm{C}$, sem aeração.

\section{Discussão}

Os fatores ambientais atuam em sinergia como responsáveis pelo crescimento, a morfogênese e a reprodução das algas marinhas bentônicas (DeBoer 1981, Lobban et al. 1985, Lobban \& Harrison 1997, Reis \& Yoneshigue-Valentin 1998, Reis \& Yoneshigue-Valentin 2000). Neste estudo destacou-se por ordem decrescente de importância o enriquecimento, a aeração, a temperatura e por fim as duas épocas do ano. No entanto, esses fatores junto com a irradiância atuam de maneira coordenada para a captação do nitrogênio (DeBoer 1981, Lobban et al. 1985).

O meio enriquecido de Provasoli (1968) mostrou eficiência no crescimento de Hypnea musciformis. Nas incubações enriquecidas (CE), com aeração (CA) e nas temperaturas de 20 e $25{ }^{\circ} \mathrm{C}$, os talos de Hypnea musciformis se mantiveram na fase exponencial, provavelmente devido a troca do meio a cada 10 dias. Além disso, a irradiância afeta a assimilação dos nutrientes diretamente através das atividades fotossintéticas (Lobban \& Harrison 1997). Segundo Dring (1982), estudos de composição dos pigmentos das algas cultivadas em laboratório, usando diferentes valores de irradiâncias, mostraram aumento na clorofila $a$ e mudança nos pigmentos acessórios em baixa irradiância. Por outro lado, em incubações sem o enriquecimento (SE), os talos desta macroalga, embora apresentando cor amarelada continuaram a crescer, porém de maneira pouco evidente (figuras 1B, 2B). Desse fato, suspeita-se que a disponibilidade de nutrientes existente na água do mar natural com origem nas águas de ressurgência de Arraial do Cabo e estocados no talo tenha sido suficiente para promover o crescimento de $H$. musciformis no curto período de 40 dias. Aliado a esse fato, a limitação do nitrogênio pode ser a causa do catabolismo de ficoeritrina, considerada uma reserva de nitrogênio para alga, reduzindo a habilidade de captar luz (Lobban \& Harrison 1997). Os resultados obtidos relacionados com a pigmentação desta planta em cultivo, serão objetos de um próximo trabalho.

Nos experimentos realizados, a aeração foi o segundo fator mais importante. Fragmentos incubados sob melhores condições $\left(\mathrm{CE}+\mathrm{CA}\right.$ e a $\left.25^{\circ} \mathrm{C}\right)$ tiveram mais sucesso do que aqueles cultivados em meio estagnado, os quais deterioraram-se após 20 dias de

Tabela 2. Resultados da análise de variância multifatorial dos dados obtidos nas diferentes condições de cultivo e nas duas épocas do ano.

\begin{tabular}{lcrcrrr}
\hline Efeito & Efeito df & Efeito MS & Erro df & Erro MS & F & Nível p \\
\hline Época do ano & 2 & 0,91705 & 48 & 0,001782 & 514,64 & 0,000001 \\
Enriquecimento & 1 & 52,59961 & 48 & 0,001782 & 29518,10 & 0,000001 \\
Temperatura & 1 & 2,56134 & 48 & 0,001782 & 1437,48 & 0,000001 \\
Aeração & 2 & 35,98347 & 48 & 0,001782 & 20193,38 & 0,000001 \\
\hline
\end{tabular}


incubação. A baixa solubilidade e difusão dos nutrientes em meio não aerado pode ter sido a causa da degeneração desses fragmentos (figura 1A) devido a formação de um filme ao redor do talo impedindo a captação dos nutrientes (Hsiao \& Druehl 1973). Embora não se tenha medido o fluxo da aeração no presente estudo, seus resultados corroboram aqueles obtidos por Reis \& Yoneshigue-Valentin (1998) e Berchez et al. (1993) onde o movimento da água foi um fator determinante na produção de biomassa de H. musciformis em populações naturais e em cultivos in situ, respectivamente. Fatores como a sedimentação e arranque das plantas dos cultivos ou do costão, no ambiente natural, vão depender do grau de exposição às ondas. As plantas de H. musciformis deste estudo foram coletadas na franja do infralitoral, num local moderadamente exposto, onde talos das populações naturais estavam cobertas por sedimentos no mês de abril.

Hierarquicamente, a temperatura foi o terceiro fator neste estudo. É bem conhecido que ela atua como regulador no padrão de distribuição das algas marinhas no contexto latitudinal (Edwards \& Kapraun 1973, Yoneshigue 1985, Faccini \& Berchez 2000). Em escala macroscópica, H. musciformis tem extensa distribuição geográfica ao longo do litoral brasileiro sob uma ampla variação de temperatura, ocorrendo nos costões rochosos do Maranhão até o Rio Grande do Sul (Schenkman 1989; Reis \& Yoneshigue-Valentin 1998). Essa rodofícea é comum nos costões rochosos influenciados pelas baixas temperaturas das águas de ressurgência (Yoneshigue 1985), de onde as plantas foram coletadas. Observa-se melhor crescimento da massa algácea a $25^{\circ} \mathrm{C}$, o que concorda com os dados de Yokoya \& Oliveira (1992b), de que os picos de crescimento dessa espécie ocorrem em altas temperaturas. Porém, H. musciformis desenvolveu-se bem em incubações submetidas tanto a $20{ }^{\circ} \mathrm{C}$ como a $25^{\circ} \mathrm{C}$, o que vem confirmar o seu caráter euritérmico (Schenkman 1989, Reis \& Yoneshigue-Valentin 1998).

Por fim, os talos da alga se aclimataram bem às condições de cultivo submetidas, embora com diferenças quanto ao ganho de massa úmida de acordo com a época de coleta (abril e julho), resultados que refletem a sazonalidade das algas coletadas em Arraial do Cabo (Yoneshigue-Valentin \& Valentin 1992). Por outro lado, estudos sobre a produção de material algáceo de três populações naturais de H. musciformis, coletadas ao longo do estado do Rio de Janeiro, demonstraram que não houve um padrão sazonal comum (Reis \& Yoneshigue-Valentin 1998), o mesmo encontrado para as plantas do litoral norte do estado de São Paulo (Faccini \& Berchez 2000).

Apesar de não terem sido efetuados testes com a irradiância, o nível utilizado de $30 \pm 5 \mu \mathrm{mol} . \mathrm{m}^{-2} . \mathrm{s}^{-1}$ foi suficiente para promover o desenvolvimento dos fragmentos de $H$. musciformis coletados no ambiente natural. Essa espécie cresce sob uma larga faixa de irradiância, até $1.800 \mu \mathrm{mol} \cdot \mathrm{m}^{-2} . \mathrm{s}^{-1}$, em dia ensolarado (Y. Yoneshigue-Valentin, dados não publicados), mas nunca menor que $30 \mu \mathrm{mol} . \mathrm{m}^{-2} \cdot \mathrm{s}^{-1}$, usado nas condições de cultivo. Esse fato vem confirmar outras observações de características intrínsecas das macroalgas, principalmente as rodofíceas, de suportarem bem as condições extremas de irradiância, tanto em qualidade como em quantidade, e por isso serem capazes de viver a profundidades, onde existe pouca luz (Yoneshigue-Valentin et al. 1995).

O controle da variação dos fatores abióticos usados neste trabalho facilitaram a interpretação de vários processos biológicos, como aqueles ligados ao crescimento de $H$. musciformis. Além disso, as condições de cultivo foram uniformes, enquanto que no ambiente natural, incluindo a região de ressurgência de Arraial do Cabo, as flutuações são mais amplas e imprevisíveis (Lobban \& Harrison 1997).

Agradecimentos - Expressamos a nossa gratidão à Capes pela bolsa de Mestrado concedida ao primeiro autor, ao Pronex 661.421/1996 - 1 que subvencionou parte deste estudo e ao CNPq pela Bolsa de Produtividade concedida ao último autor (proc. Al. 521.783/96-8). Ao prof. Jean L. Valentin pelas críticas e sugestões quanto a análise estatística. Agradecemos também aos revisores anônimos pelas valiosas contribuições para melhoria deste trabalho.

\section{Referências bibliográficas}

BERCHEZ, F.A.S. \& OLIVEIRA, E.C. 1989. Maricultural essays with carrageenophyte Hypnea musciformis in S. Paulo, Brazil. In Cultivation of seaweed in Latin America (E.C. Oliveira-Filho \& N. Kautsky, eds.). Universidade de São Paulo, São Paulo, p.89-94.

BERCHEZ, F.A.S., PEREIRA, R.T.L. \& MARQUES, H.L. 1989. A influência da predação na maricultura de Hypnea musciformis (Rhodophyta, Gigartinales). Ínsula 19:71-82.

BERCHEZ, F.A.S., PEREIRA, R.T.L. \& KAMIYA, N.F. 1993. Culture of Hypnea musciformis (Rhodophyta, Gigartinales) on artificial substrates attached to linear ropes. Hydrobiologia 260/261:415-420.

DEBOER, J.A. 1981. Nutrients. In The biology of seaweeds (C.S. Lobban \& M.J. Wynne, eds.). Blackwell Scientific Publications, Oxford, p.356-391. 
DRING, M.J. 1982. The biology of marine plants (Contemporary biology). Edward Arnold Publishers Ltd., Great Britain.

DURAKO, M.J. \& DAWES, C.J. 1980. A comparative study of two populations of Hypnea musciformis from the east and west coasts of Florida, USA. II. Photosynthetic and respiratory rates. Marine Biology 59:157-162.

FACCINI, A.L. \& BERCHEZ, F. 2000. Management of natural beds and standing stock evaluation of Hypnea musciformis (Gigartinales, Rhodophyta) in south-eastern Brazil. Journal of Applied Phycology 12:101-103.

EDWARDS, P. \& KAPRAUN, D.F. 1973. Benthic marine algae ecology in the Port Arkansas, Texas area. Contribution in Marine Science 17:15-52.

HSIAO, S.I.C. \& DRUEHL, L.D. 1973. Environmental control of gametogenesis in Laminaria saccharina. IV. In situ development of gametophytes and young sporophytes. Journal of Phycology 9:160-164.

LOBBAN, C.S., HARRISON, P.J. \& DUNCAN, M.J. 1985. The physiological ecology of seaweeds. Cambridge University Press, Cambridge.

LOBBAN, C.S. \& HARRISON, P. J. 1997. Seaweeds ecology and physiology. Cambridge University Press, Cambridge.

MSHIGENI, K.E. 1976. Field cultivation of Hypnea (Rhodophyta) spores for carrageenan: prospects and problems. Botanica Marina 19:227-230.

MSHIGENI, K.E. 1977. Seasonal changes in the standing crops of three Hypnea species (Rhodophyta, Gigartinales) in Hawai. Botanica Marina 20:303-306.

OLIVEIRA FILHO, E.C. 1981. Marine Phycology and exploitation of seaweeds in South America. In Proceedings of $X^{\text {th }}$ International Seaweed Symposium (T. Levring, ed.). Walter de Gruyter, New York, v.10, p.97-112.

OLIVEIRA FILHO, E.C. 1998. The seaweeds resources of Brazil. In Seaweeds resources of the world (A.T. Critchley \& M. Ohno, eds.). Japan International Cooperation Agency, Yokosuka, p.366-371.

PROVASOLI, L. 1968. Media and prospects for cultivation of marine algae. In Cultures and Collections of Algae (A. Watanabe \& A. Hattori, eds.). Japanese Society of Plant Physiology, Tokyo, p. 47-74.

RAO, R.K. 1970. Studies on growth cycle and phycocolloid content in Hypnea musciformis (Wulf) Lamour. Botanica Marina 13:163-167.
REIS, R.P. \& YONESHIGUE-VALENTIN, Y. 1998. Variação espaço-temporal de populações de Hypnea musciformis (Rhodophyta, Gigartinales) na Baía de Sepetiba e Armação de Búzios, R.J., Brasil. Acta Botânica Brasílica 12:465-483.

REIS R.P. \& YONESHIGUE-VALENTIN, Y. 2000. Phenology of Hypnea musciformis (Wulfen) Lamouroux (Rhodophyta, Gigartinales) in three populations from Rio de Janeiro State, Brazil. Botanica Marina 43:299-304.

SANTOS, M.G.M., LAGROTA, M.H.C., MIRANDA, M.F.S., YONESHIGUE-VALENTIN, Y. \& WIGG, M.D. 1999. A screening for antiviral effect of extracts from Brazilian marine algae against acyclovir resistant Herpes simplex virus type 1. Botanica Marina 42:227-230.

SCHENKMAN, R.P.F. 1989. Hypnea musciformis (Rhodophyta) - ecological influence on growth. Journal of Phycology 25:192-196.

WALLNER, M., LOBO, S., BOCCANERA, N. \& SILVA, E.M. 1992. Biomass, carrageenan yield and reproductive state of Hypnea musciformis (Rhodophyta, Gigartinales) under natural and experimental cultivated condition. Aquaculture and Fisheries Management 23:443-451.

YOKOYA, N.S. \& OLIVEIRA, E.C. 1992a. Effects of salinity on the growth rate, morphology and water content of some Brazilian red algae of economic importance. Ciencias Marinas 18:49-64.

YOKOYA, N.S. \& OLIVEIRA, E.C. 1992b. Temperature responses of economically important red algae and their potential for mariculture in Brazilian waters. Journal of Applied Phycology 4:339-345.

YONESHIGUE, Y. 1985. Taxonomie et ecologie des algues marines dans la région de Cabo Frio (Rio de Janeiro, Brésil). These d'Etat Science, Faculté des Sciences de Luminy, Marseille.

YONESHIGUE-BRAGA, Y. \& NEVES, M.H.C.B. 1981. Preliminary studies on mass culture of Gracilaria sp. using different nutrient media. In Proceedings of $\mathrm{X}^{\text {th }}$ International Seaweed Symposium. (T. Levring, ed.). Walter de Gruyter, New York, v.10, p.643-648.

YONESHIGUE-VALENTIN, Y., MITCHELL, G.J.P. \& GURGEL, C.F.D. 1995. Quelques observations preliminaires sur les macroalgues de la plate forme continentale du sud est brésilien. Acta Botanica Gallica 142:161-165.

YONESHIGUE-VALENTIN, Y. \& VALENTIN, J.L. 1992. Macroalgae of Cabo Frio upwelling region, Brazil: ordination of communities. In Coastal Plant Communities of Latin America (U. Seeliger, ed.). Academic Press, New York, p.31-49. 